\title{
Fabrication of Single-Layer Touch Screen Panel with Corrosion Resistant Metal-Mesh Electrodes
}

\author{
Kyoung Soo Chae, ${ }^{1,2}$ Sung Han, ${ }^{2}$ Jae Youn Jeong, ${ }^{2}$ and Tae Hee Han ${ }^{1 *}$ \\ ${ }^{1}$ College of Information and Communication Engineering, Sungkyunkwan University, \\ 2066 Seobu, Jangan, Suwon, Gyeonggi 16419, Korea \\ ${ }^{2}$ Samsung Electro-Mechanics Co., 150 Maeyoung, Yeongtong, Suwon, Gyeonggi 16674, Korea \\ (Received April 27, 2017; accepted May 18, 2017)
}

Keywords: touch screen panel, metal-mesh, corrosion, transparent, sheet resistance

We demonstrate a 7-inch single-layer touch screen panel (TSP) using highly conductive, flexible, and transparent metal-mesh electrodes. Our touch sensor shows a transmittance of $86.3 \%$ at $550 \mathrm{~nm}$ and high corrosion resistance in $85 / 85$ and salt spray testing. The $85 / 85$ test was chosen to evaluate the combined effects of high humidity and high temperature. The combined stress of $85^{\circ} \mathrm{C}$ and $85 \%$ relative humidity (RH) did not cause failure in the TSP.

\section{Introduction}

Touch sensors on many IT devices such as smartphones, tablets, and monitors are manufactured using indium tin oxide (ITO), which has high light transmittance ${ }^{(1)}$ and no pattern visibility. However, one limitation of ITO is the requirement of a large area due to its high surface resistance. Additionally, a lack of physical flexibility makes it difficult to use in flexible displays. As alternatives, copper or silver-based metal meshes with high conductivity and excellent flexibility have been developed and actively studied. ${ }^{(2-4)}$ To commercialize an opaque metal electrode using a touch sensor, it is necessary to improve the transmittance and to form a pattern that cannot be recognized. In addition, it is necessary to overcome its sensitivity to the heat and corrosive chemicals used in conventional photolithography processes.

The transmittance can be increased by decreasing the mesh line width and increasing the pitch as shown in Fig. $1 .^{(5)}$ The visibility can be improved by a black-oxide treatment to reduce the amount of light reflected from the metal surface. ${ }^{(3)}$ However, the results of studies on improving

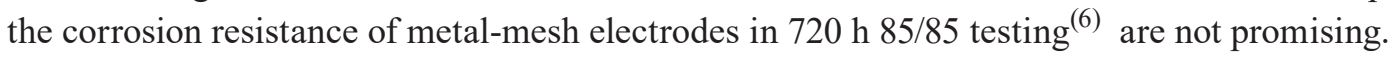

In this study, we developed a single layer 7-inch touch screen panel (TSP) with high corrosion resistance. The causes of corrosion in metal-mesh electrodes and bus lines of the passivation/ conduction/passivation (PCP) structure ${ }^{(7)}$ used to improve the corrosion resistance were analyzed. Based on the analytical results, an experiment was then conducted to determine the optimal thickness of the top passivation layer to improve the corrosion resistance of the metal-mesh electrodes. Using a metal layer with the optimized PCP structure, a TSP could be fabricated that is corrosion free after being subjected to a $720 \mathrm{~h} 85 / 85$ test.

${ }^{*}$ Corresponding author: e-mail: than@skku.edu http://dx.doi.org/10.18494/SAM.2017.1633 


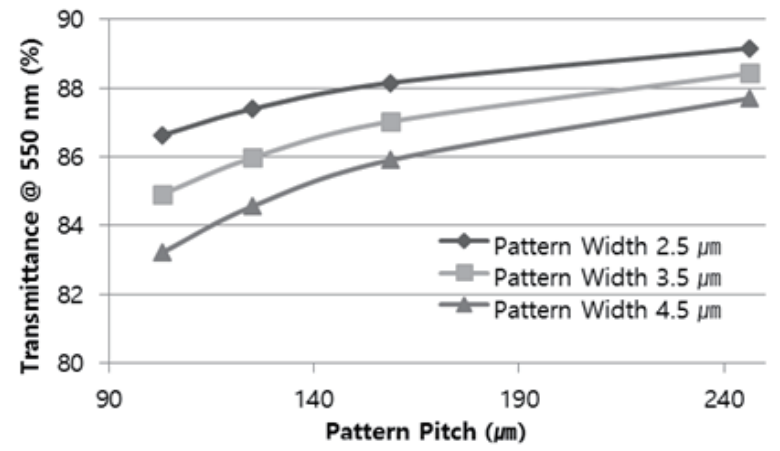

Fig. 1. Variation of transmittance of metal mesh sensors with respect to pattern density.

\section{Fabrication of Single-Layer TSP}

We fabricated a 7-inch single-layer TSP using metal-mesh electrodes on a flexible substrate (Fig. 2). The metal-mesh electrodes were deposited as PCP structures. The TSP was then evaluated for corrosion resistance and for its optical and electrical properties. The touch sensor for the TSP was fabricated by an $\mathrm{R} 2 \mathrm{R}$ process consisting of the following steps: sputtering, lamination, exposure, development/etching/strip (DES), and inspection. Figure 3 illustrates the R2R process. To produce the PCP structure, a $20 \mathrm{~nm}$-thick Cu-Ni alloy layer is first deposited on PET film. After sputtering the bottom alloy layer, a $100 \mathrm{~nm}$-thick $\mathrm{Cu}$ layer is sputtered on the bottom alloy layer. Then, varying thicknesses of a top $\mathrm{Cu}-\mathrm{Ni}$ alloy layer are deposited on the $\mathrm{Cu}$ layer. After the deposition process, the metal layer is patterned using post processing, and a flexible printed circuit board (FPCB) is attached to the pattern using anisotropic conductive film (ACF).

\section{Failure Analysis in Environmental Reliability Test}

Figure 4 shows the results of cross-sectional analysis of a corroded region from the 85/85 test. As a result of the enlargement of the pore area shown in the figure, it was found that the inner conduction layer was corroded and only the top passivation layer remained. In the transmission electron microscope (TEM)-bright field (BF) image, a small hole in the top passivation layer was detected, and it was confirmed that there was corrosion in a wide area of the lower conduction layer. From these results, it was concluded that typical pitting corrosion ${ }^{(8)}$ occurred.

Among the many causes of metal corrosion, pitting corrosion refers to rapid corrosion that progresses inward through the protective layer of a surface. It does not occur in areas where widespread corrosion is present. This corrosion characteristic is consistent with the TEM image observed, especially where the uppermost passivation layer is perforated and the corrosion of the internal conduction layer is relatively intense. Pitting corrosion is a major failure mode in $85 / 85$ testing.

In addition to these observations, elemental analysis using a scanning transmission electron microscope (STEM) was performed on the area where pitting corrosion was observed. As shown in Fig. 5, $\mathrm{Cl}^{-}$ions were present in the area where the pore exhibited pitting corrosion and absent in the areas that were not corroded. This result is observed on both the right and left lower images of Fig. 5. This clearly indicates that $\mathrm{Cl}^{-}$ions play an important role in the pitting corrosion. 


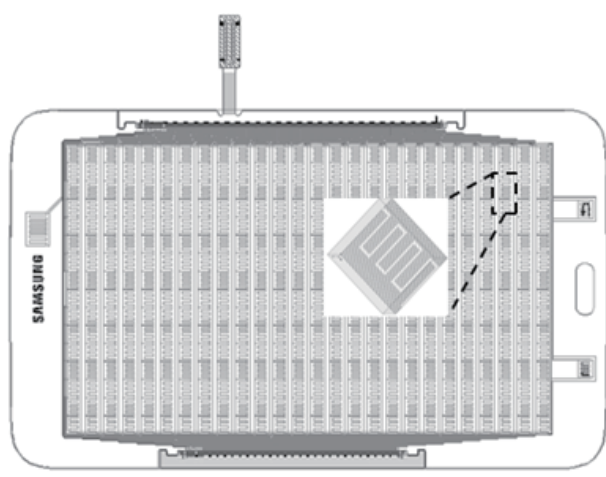

(a)

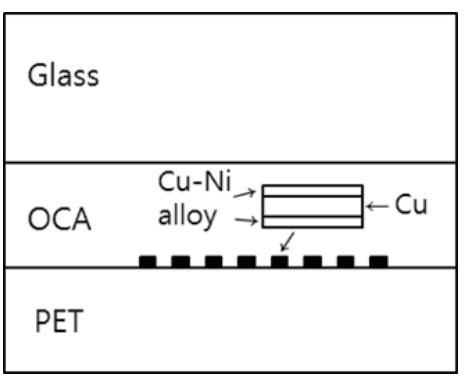

(b)

Fig. 2. An overview of the proposed TSP: (a) schematic and (b) structure.

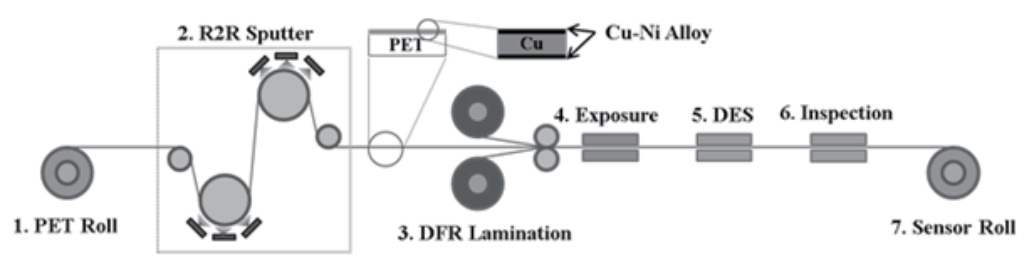

Fig. 3. R2R process used for the fabrication of touch sensors.

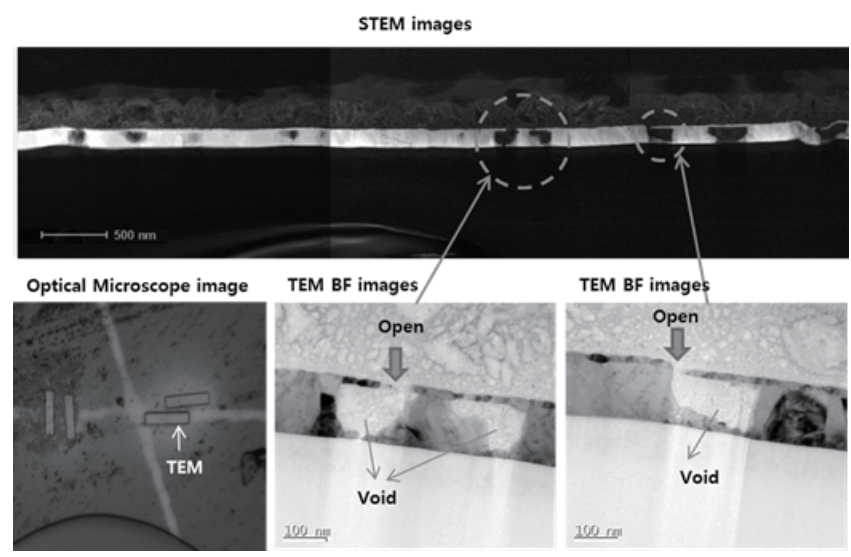

Fig. 4. Results of TEM section analysis in defective area.

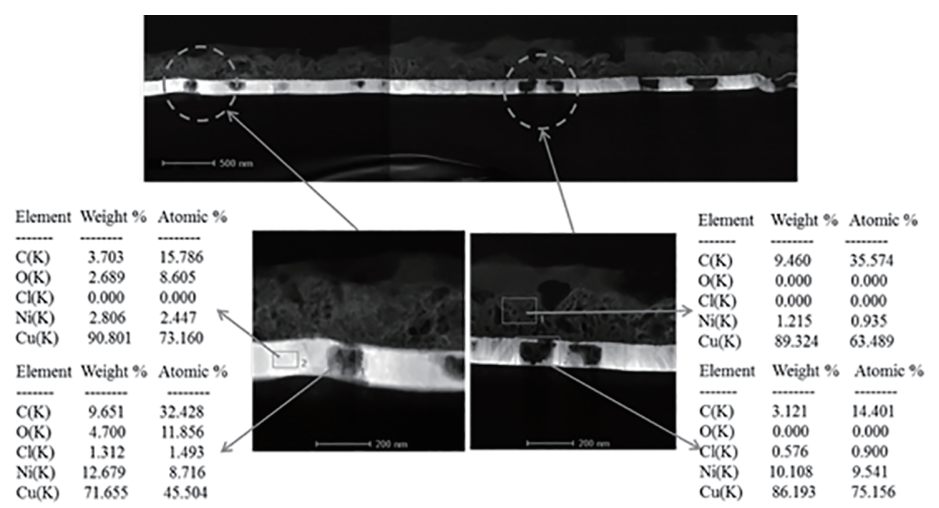

Fig. 5. Results of energy dispersive X-ray analysis (EDX) in the defective areas and surrounding areas. 
From this analysis, it was concluded that pitting corrosion proceeds rapidly through the surface of the top passivation layer damaged by dissociated $\mathrm{Cl}^{-}$ions which react with moisture. Therefore, we chose to increase the thickness of the top passivation layer, which acts as a barrier, to improve the resistance to pitting corrosion. A barrier film ${ }^{(9,10)}$ can be applied to prevent penetration of moisture into the passivation layer, but this was not done in this study due to the mechanical problems associated with increasing the ultimate thickness of the TSP.

To verify the integrity of the passivation layer, the pitting corrosion resistance to salt spray was evaluated by increasing the deposition thickness of the top passivation layer from $20 \mathrm{~nm}$ to 30,40 , and $60 \mathrm{~nm}$. The salt water spray consists of a $5 \mathrm{wt} \%$ aqueous solution of $\mathrm{NaCl}$, and the TSP was kept in an accelerated corrosive environment where oxygen, $\mathrm{Cl}^{-}$ions, and moisture were continuously supplied. The passivation layer was then cleaned with pure water. A sample dried at room temperature with an air blower was then observed under an optical microscope. For a top passivation layer thickness of $20 \mathrm{~nm}$, it was confirmed that pitting corrosion occurred over the entire surface within $5 \mathrm{~min}$. As the passivation thickness was increased, the frequency and density of pitting corrosion decreased. Therefore, it was confirmed that increasing the thickness of the top passivation layer delays damage due to pitting corrosion. Figures 6(a) and 6(b) show the surface of the top passivation layer and the sheet resistance of the thin metal film of the PCP structure versus exposure time to salt spray.

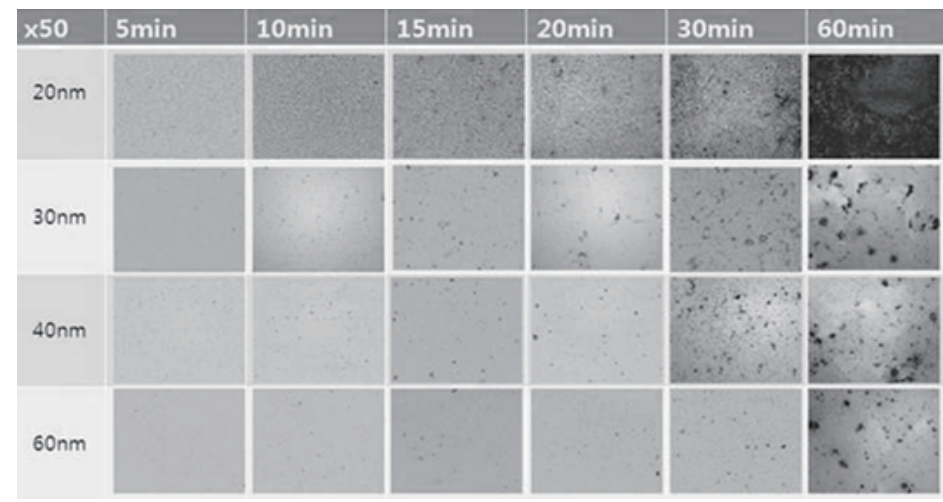

(a)

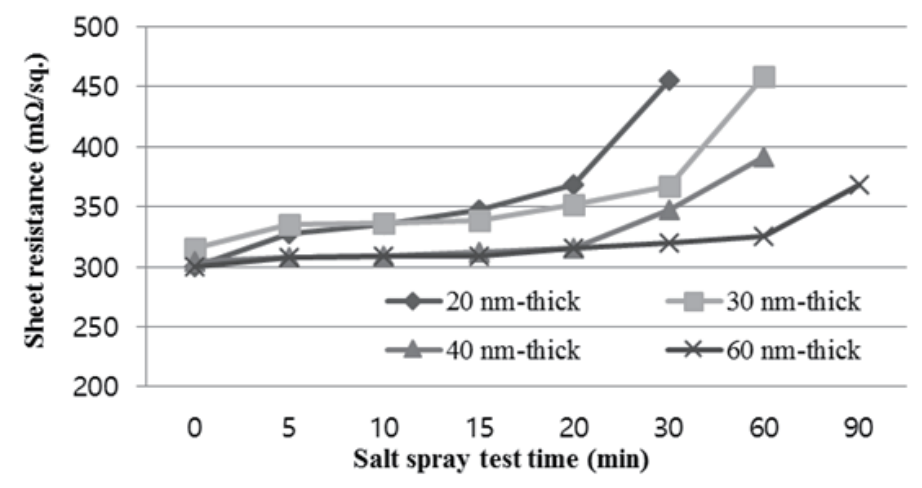

(b)

Fig. 6. Time dependences of (a) the state of surface and (b) sheet resistance after spraying with 5 wt $\%$ aqueous $\mathrm{NaCl}$. 


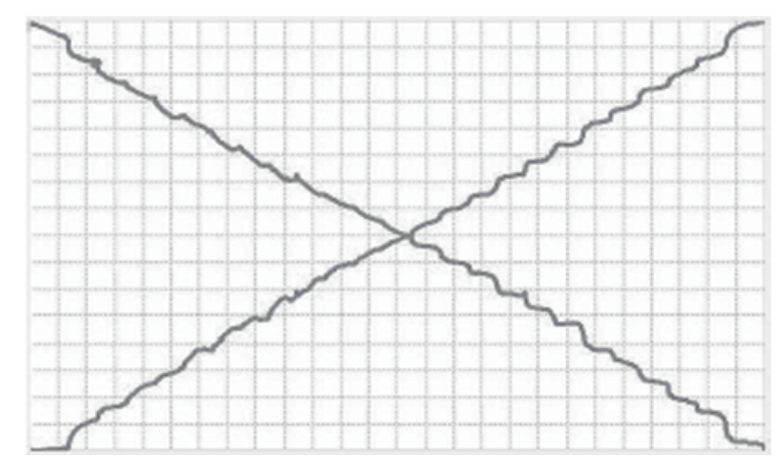

Fig. 7. Linearity results for a single layer 7-inch TSP with $4 \Phi$ cooper stick.

\section{Results}

\subsection{The 85/85 test of single-layer TSP}

The steady-state temperature humidity bias life test is known as the $85 / 85$ test. The temperature is held at a constant $85{ }^{\circ} \mathrm{C}$ and at a $\mathrm{RH}$ of $85 \%$, and the test "is performed for the purpose of evaluating the reliability of non-hermetic packaged solid-state devices in humid environments. It employs conditions of temperature, humidity, and bias that accelerate the penetration of moisture through the external protective material or along the interface between the external protective material and the metallic conductors which pass through it". ${ }^{(6)}$

The TSP was fabricated on thin metal film with a top passivation layer deposited at various thicknesses and the 85/85 test was performed for $720 \mathrm{~h}$. Only the TSP with a PCP structure of metal-mesh electrodes with a top passivation layer thickness of $60 \mathrm{~nm}$ had no failures. For the 60 -nm-thick passivation layer, it is clear that the combined conditions of $85{ }^{\circ} \mathrm{C}$ and $85 \% \mathrm{RH}$ did not constitute a high enough stress to cause the TSP to fail.

\subsection{Optical, electrical, and operational properties of single-layer TSP}

The optical transmittance and the sheet resistance of the samples were investigated. Throughout the optimization of deposition conditions, we obtained a transmittance value of $86.3 \%$ at a wavelength of $550 \mathrm{~nm}$ and a sheet resistance of $40 \Omega / \mathrm{sq}$.

After attaching the TSP to a smartphone, a touch operation test was performed with a $4 \Phi$ stylus. As shown in Fig. 7, we confirmed that the TSP works normally in terms of touch sensitivity. The linearity, however, is poor. This will be improved later with a modified touch electrode pattern.

\section{Conclusion}

We have developed a single layer 7-inch TSP using a highly conductive and transparent PCP electrode. A touch sensor with a metal-mesh PCP structure was fabricated using an R2R process. The linewidth of the fabricated electrode was $3.5 \mu \mathrm{m}$, the sheet resistance was $40 \Omega / \mathrm{sq}$, and the transmittance was $86.3 \%$ at $550 \mathrm{~nm}$. To strengthen the corrosion resistance, the main causes of 
corrosion were analyzed. Based on the results, the thickness of the top passivation layer was optimized and the TSP was able to withstand the $720 \mathrm{~h} 85 / 85$ test.

\section{Acknowledgments}

This research was supported by the Basic Science Research Program through the National Research Foundation of Korea (NRF) funded by Ministry of Education (NRF2015R1D1A1A01057278).

\section{References}

1 S.-C. Her and C.-F. Chang: Sens. Mater. 28 (2016) 975.

2 H. S. Kim, S. J. Yang, K. J. Noh, and S. E. Lee: J. Korean Inst. Electr. Electron. Mater. Eng. 29 (2016) 414.

3 S. Son, J. E. Park, J. Lee, M. Yang, and B. Kang: Homepage of Scientific Reports, https://www.nature.com/ articles/srep34629 (accessed 2016).

4 Y. Choi, K. Kim, E. and T. Lee: SID 2014 DIGEST (SID, San Diego, 2014) pp. 197-199.

5 S. M. Bergin, Y. H. Chen, A. R. Rathmell, P. Charbonneau, Z. Y. Li, and B. J. Wiley: Nanoscale 4 (2012) 1996.

6 J. Virki and P. Raumonen: Act. Passive Electron. Compon. 2011 (2011) 1.

7 S. Kwak, M. Kwak, B. Ju, and S. Hong: J. Nanosci. Nanotechnol. 15 (2015) 7645.

8 P. R. Roberge: Homepage of Corrosion Doctors, http://www.corrosion-doctors.org/Forms-pitting/Pitting.htm (accessed December 2016).

9 i-componets Co.: Homepage of i-Barrier i-Metalish catalogue, http://www.i-components.co.kr/pdf/i-Barrier i-Metalish_catalogue.pdf (accessed December 2016).

10 Vitriflex Inc.: Ultra-Barrier films, http://www.vitriflex.com (accessed December 2016). 